\title{
Ältere profitieren von Androgentherapie
}

Die Androgenbehandlung mit dem niedrig dosierten Anabolikum Norethandrolon als Erhaltungstherapie verlängert bei älteren Patienten (median 70 Jahre) mit akuter myeloischer Leukämie (AML) die Zeit bis zur Krankheitsprogression sowie die Gesamtüberlebenszeit. Arnaud Pigneux und seine Kollegen beobachteten dies in der prospektiven offenen Studie LAM-SA2002, an der mehr als 300 Patienten teilnahmen. Die Patienten waren mindestens 60 Jahre alt und hatten einen ECOG-Performancestatus von höchstens 2 (von maximal 5 Stufen) sowie eine Lebenserwartung von mindestens einem Monat.

Die Induktionstherapie bestand aus der Applikation von Idarubicin, Cytarabin und Lomustin (CCNU). Über zwei Jahre folgte danach alle drei Monate jeweils ein weiterer Zyklus. Zwischen den Zyklen erhielten die Patienten als Erhal-

\section{kurz notiert}

\section{Kampf gegen Strahlenresistenz}

Eine mögliche Strategie zur Überwindung einer Strahlenresistenz oraler Plattenepithelkarzinome haben japanische Forscher kürzlich entdeckt. In ihren Untersuchungen konnten sie zeigen, dass erhöhte Interleukin-6(IL-6)-Spiegel den strahleninduzierten Zelltod von Krebszellen unterdrücken. Dabei schützt IL-6 die Krebszellen durch Aktivierung des Downstream-Moleküls STAT3 sowie durch Interaktion mit dem antioxidativen Nrf2-Signalweg vor den Folgen der Bestrahlung. Diese Effekte führen zu einer signifikanten Reduktion von oxidativem Stress und einer verminderten strahleninduzierten DNA-Schädigung. Für ihre Untersuchungen verwendeten die Wissenschaftler Tumorgewebe von Patienten mit strahlenresistenten fortgeschrittenen oralen Plattenepithelkarzinomen sowie humane Zellkulturen. Nun hoffen sie, dass neue Therapien, die auf IL-6 abzielen, bei strahlenresistenten Tumoren vorteilhaft sind und das Ansprechen auf die Bestrahlung verbessern. Im Mausmodell konnten sie bereits zeigen, dass die Blockade des IL-6-Signalwegs durch das Immunsuppressivum Tocilizumab, das derzeit zur Behandlung der rheumatoiden Arthritis eingesetzt wird, Krebszellen gegenüber einer Strahlentherapie empfindlicher macht [Matsuoka Y et al. Brit J Cancer. 2016; 115(10):1234-44]. Judith Neumaier tungstherapie Mercaptopurin und Methotrexat. 247 Patienten mit einer kompletten bzw. partiellen Remission nach der antineoplastischen Behandlung erhielten über zwei Jahre eine Erhaltungstherapie mit niedrig dosiertem Norethandrolon oder keine weitere Therapie.

In der Gruppe mit Androgentherapie lebten nach fünf Jahren noch 31,2\% der Patienten mit kompletter Remission, in der Vergleichsgruppe ohne diese Behandlung waren nur noch $16,2 \%$ ohne Krankheitszeichen. Ein Nutzen dieser Erhaltung wurde frühestens nach einem Jahr beobachtet. Die ereignisfreie Überlebensrate nach fünf Jahren war in der Gruppe mit Androgenbehandlung ebenfalls deutlich höher (21,5 vs. 12,9\%). Selbst beim Gesamtüberleben gab es einen signifikanten Unterschied zwischen den beiden Studienarmen (26,3 vs. 17,2 \%; $\mathrm{p}=0,008)$. In einer weiteren Analyse

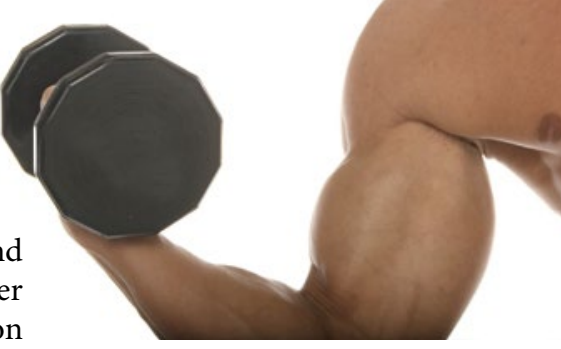

Von Anabolika profitieren nicht nur die Muskeln, sondern auch AML-Patienten.

stellte sich heraus, dass der Nutzen der Androgentherapie nur bei jenen Patienten $z u$ verzeichnen war, deren Leukozytenzahl nicht über $30 \times 10^{9} / 1 \mathrm{lag}$.

Bei den Nebenwirkungen gab es jedoch keine Unterschiede zwischen den beiden Studienarmen. Möglicherweise werde durch Norethandrolon unter anderem die Proliferation verbliebener Blasten unterdrückt, so die Autoren. PeterLeiner

Pigneux A et al. Addition of Androgens Improves Survival in Elderly Patients With Acute Myeloid Leukemia: A GOELAMS Study. J Clin Oncol. 2017; 35(4):387-393.
Bei Patienten mit ALK-positivem nichtkleinzelligem Lungenkarzinom (NSCLC), die mit Crizotinib vorbehandelt wurden, zeigt Alectinib eine gute Wirksamkeit gegen ZNS-Metastasen, zusätzlich zur systemischen Aktivität. Das ergab eine gemeinsame Auswertung der beiden einarmigen Phase-II-Studien NP28761 und NP28673. An diesen nahmen insgesamt 225 Patienten mit ALK-positivem NSCLC teil, die mit Crizotinib vorbehandelt waren. Alle Patienten erhielten zweimal täglich $600 \mathrm{mg}$ Alectinib. Der primäre Endpunkt war jeweils die objektive Ansprechrate. 136 Patienten hatten zu Studienbeginn Metastasen im ZNS (60\%), 50 Patienten (37\%) eine nachweisbare ZNSErkrankung. 95 Patienten (70\%) hatten vorher eine Bestrahlung des ZNS erhalten. 55 Patienten beendeten die ZNS-Radiotherapie mehr als sechs Monate vor dem Beginn mit Alectinib.

Die mediane Beobachtungsdauer betrug 12,4 Monate. Bei Patienten mit nachweisbarer ZNS-Erkrankung lag die ob- jektive Ansprechrate im ZNS bei 64,0\%, wobei $11 \%$ ein vollständiges Ansprechen zeigten. Die Rate der Krankheitskontrolle im ZNS betrug 90,0\% und die mediane Dauer des Ansprechens im ZNS 10,8 Monate. Bei Patienten mit messbarer und/oder nicht messbarer ZNS-Erkrankung betrug die objektive Ansprechrate im ZNS 42,6\% (vollständiges Ansprechen: $27 \%$ ), die Rate der Krankheitskontrolle im ZNS 85,3\% und die mediane Dauer des Ansprechens im ZNS 11,1 Monate. Bei Patienten mit vorhergehender Bestrahlung $(n=95)$ betrug die objektive Ansprechrate im ZNS 35,8\% und bei Patienten ohne vorherige Bestrahlung ( $\mathrm{n}=41$ ) $58,5 \%$. Bis zum Zeitpunkt der Auswertung blieben $61 \%$ der Patienten mit ZNS-Metastasen progressionsfrei.

Judith Neumaier

Gadgeel SM et al. Pooled Analysis of CNS Response to Alectinib in Two Studies of Pretreated Patients With ALK-Positive Non-Small-Cell Lung Cancer. J Clin Oncol. 2016;34(34):4079-85. 